\title{
Asymptotics of the Eigenvalues of a Self-Adjoint Fourth Order Boundary Value Problem with Four Eigenvalue Parameter Dependent Boundary Conditions
}

\author{
Manfred Möller and Bertin Zinsou \\ The John Knopfmacher Centre for Applicable Analysis and Number Theory, School of Mathematics, \\ University of the Witwatersrand (Wits), Private Bag 3, Johannesburg 2050, South Africa
}

Correspondence should be addressed to Manfred Möller; manfred.moller@wits.ac.za

Received 9 April 2013; Revised 24 May 2013; Accepted 27 May 2013

Academic Editor: Janusz Matkowski

Copyright (C) 2013 M. Möller and B. Zinsou. This is an open access article distributed under the Creative Commons Attribution License, which permits unrestricted use, distribution, and reproduction in any medium, provided the original work is properly cited.

Considered is a regular fourth order ordinary differential equation which depends quadratically on the eigenvalue parameter $\lambda$ and which has separable boundary conditions depending linearly on $\lambda$. It is shown that the eigenvalues lie in the closed upper half plane or on the imaginary axis and are symmetric with respect to the imaginary axis. The first four terms in the asymptotic expansion of the eigenvalues are provided.

\section{Introduction}

Sturm-Liouville problems have attracted extensive attention due to their intrinsic mathematical challenges and their applications in physics and engineering. Classical Sturm-Liouville problems have been extended to higher-order differential equations and to differential equations with eigenvalue parameter dependent boundary conditions. For example, the generalized Regge problem is realised by a second order differential operator which depends quadratically on the eigenvalue parameter and which has eigenvalue parameter dependent boundary conditions, see [1]. The particular feature of this problem is that the coefficient operators of this pencil are selfadjoint, and it is shown in [1] that this gives some a priori knowledge about the location of the spectrum. In [2], this approach has been extended to a fourth order differential equation describing small transversal vibrations of a homogeneous beam compressed or stretched by a force $g$. Again, this problem is represented by a quadratic operator pencil, in a suitably chosen Hilbert space, whose coefficient operators are self-adjoint. In [3], we have considered this fourth order differential equation with general two-point boundary conditions which depend linearly on the eigenvalue parameter. Necessary and sufficient conditions such that the associated operator pencil consists of self-adjoint operators have been obtained. In $[4,5]$, we have derived eigenvalue asymptotics associated with particular boundary conditions. In this paper, we are considering the case of separable boundary conditions where all four of these boundary conditions depend on the eigenvalue parameter.

Other recent results on fourth order differential operators whose boundary conditions depend on the eigenvalue parameter but which are represented by linear operator pencils, include spectral asymptotics and basis properties, see [68].

In Section 2, we introduce the operator pencil associated with the eigenvalue problem (1), (2), and we derive the boundary conditions such that the operators in the pencil are self-adjoint. In Section 3, we obtain the location of the spectrum and the asymptotic distribution of the eigenvalues for the case $g=0$. In Section 4 , we prove that the boundary value problem under investigation is Birkhoff regular, which implies that the eigenvalues for general $g$ are small perturbations of the eigenvalues for $g=0$. Hence, in Section 5, we derive the first four terms of the asymptotics of the eigenvalues and compare them to those obtained for the boundary conditions considered in [5]. 


\section{The Quadratic Operator Pencil $L$}

On the interval $[0, a]$, we consider the boundary value problem

$$
\begin{gathered}
y^{(4)}-\left(g y^{\prime}\right)^{\prime}=\lambda^{2} y, \\
B_{j}(\lambda) y=0, \quad j=1,2,3,4,
\end{gathered}
$$

where $g \in C^{1}[0, a]$ is a real valued function and (2) is separated boundary conditions with the $B_{j}$ depending linearly on the eigenvalue parameter $\lambda$. The boundary conditions (2) are taken at the endpoint 0 for $j=1,2$ and at the endpoint $a>0$ for $j=3,4$. Further, we assume for simplicity that

$$
B_{j}(\lambda) y=y^{\left[p_{j}\right]}\left(a_{j}\right)+i \alpha \varepsilon_{j} \lambda y^{\left[q_{j}\right]}\left(a_{j}\right)
$$

where $a_{j}=0$ for $j=1,2$ and $a_{j}=a$ for $j=3,4,0 \leq q_{j}<p_{j} \leq$ $3, \alpha>0$, and $\varepsilon_{j} \in \mathbb{C} \backslash\{0\}$. We recall that the quasi-derivatives associated with (1) are given by

$$
\begin{gathered}
y^{[0]}=y, \quad y^{[1]}=y^{\prime}, \quad y^{[2]}=y^{\prime \prime}, \\
y^{[3]}=y^{(3)}-g y^{\prime}, \quad y^{[4]}=y^{(4)}-\left(g y^{\prime}\right)^{\prime},
\end{gathered}
$$

see [9, page 26]. In order to have independent boundary conditions, we will also assume that the numbers $p_{1}, q_{1}, p_{2}$, $q_{2}$ as well as the numbers $p_{3}, q_{3}, p_{4}, q_{4}$ are mutually disjoint.

Recall that in applications, using separation of variables, the parameter $\lambda$ emanates from derivatives with respect to the time variable in the original partial differential equation, and it is reasonable that the highest space derivative occurs in the term without time derivative. Thus, the most relevant boundary conditions would have $q_{j}<p_{j}$ for $j=1, \ldots, 4$.

Further assumptions on the $p_{j}, q_{j}$, and $\varepsilon_{j}$ will be made later and will be justified by the requirements on the operator pencil which we are going to define now.

For rather generic boundary conditions, a quadratic operator pencil has been associated in [3], and we will now recall notations and results from [3] which are relevant in our case.

We denote by $U$ the collection of the boundary conditions (2) and define the following operators related to $U$ :

$$
\begin{array}{r}
U_{0} y=\left(y^{\left[p_{j}\right]}\left(a_{j}\right)\right)_{j=1}^{4}, \quad U_{1} y=\left(\varepsilon_{j} y^{\left[q_{j}\right]}\left(a_{j}\right)\right)_{j=1}^{4}, \\
y \in W_{4}^{2}(0, a),
\end{array}
$$

where $W_{4}^{2}(0, a)$ is the Sobolev space of order 4 on the interval $(0, a)$. We consider the linear operators $A(U), K$, and $M$ in the space $L_{2}(0, a) \oplus \mathbb{C}^{4}$ with domains

$$
\begin{gathered}
\mathscr{D}(A(U))=\left\{\tilde{y}=\left(\begin{array}{c}
y \\
U_{1} y
\end{array}\right): y \in W_{4}^{2}(0, a)\right\}, \\
\mathscr{D}(K)=\mathscr{D}(M)=L_{2}(0, a) \oplus \mathbb{C}^{4},
\end{gathered}
$$

given by

$$
\begin{gathered}
(A(U)) \tilde{y}=\left(\begin{array}{l}
y^{[4]} \\
U_{0} y
\end{array}\right) \quad \text { for } \tilde{y} \in \mathscr{D}(A(U)), \\
K=\left(\begin{array}{ll}
0 & 0 \\
0 & I
\end{array}\right), \quad M=\left(\begin{array}{ll}
I & 0 \\
0 & 0
\end{array}\right) .
\end{gathered}
$$

It is easy to check that $K \geq 0, M \geq 0, M+K=I$, and $\left.M\right|_{\mathscr{D}(A(U))}>0$. We associate a quadratic operator pencil

$$
L(\lambda, \alpha)=\lambda^{2} M-i \alpha \lambda K-A(U), \quad \lambda \in \mathbb{C},
$$

in the space $L_{2}(0, a) \oplus \mathbb{C}^{4}$ with the problem (1), (2). We observe that (8) is an operator representation of the eigenvalue problem (1), (2) in the sense that a function $y$ satisfies (1), (2) if and only if $L(\lambda, \alpha) \tilde{y}=0$.

For the boundary conditions (2) with the assumptions made so far, [3, Theorem 1.2] leads to the following.

Proposition 1. The differential operator $A(U)$ associated with (1), (2) is self-adjoint if and only if for $j=1, \ldots, 4$; the numbers $p_{j}, q_{j}$, and $\varepsilon_{j}$ satisfy the following conditions: $p_{j}+q_{j}=3, \varepsilon_{j}=$ 1 if $q_{j}$ is even in case $a_{j}=0$ or odd in case $a_{j}=a$, and $\varepsilon_{j}=-1$, otherwise.

Since we aim to consider the boundary eigenvalue problem (1), (2) in case that $A(U)$ is self-adjoint, the boundary operators are, up to permutation,

$$
\begin{aligned}
& B_{1}(\lambda) y=y^{\prime \prime}(0)-i \alpha \lambda y^{\prime}(0), \\
& B_{2}(\lambda) y=y^{[3]}(0)+i \alpha \lambda y(0), \\
& B_{3}(\lambda) y=y^{\prime \prime}(a)+i \alpha \lambda y^{\prime}(a), \\
& B_{4}(\lambda) y=y^{[3]}(a)-i \alpha \lambda y(a) .
\end{aligned}
$$

As in [4, Proposition 2.3], we obtain the following.

Proposition 2. The operator pencil $L(\cdot, \alpha)$ is a Fredholm valued operator function with index 0 . The spectrum of the Fredholm operator $L(\cdot, \alpha)$ consists of discrete eigenvalues of finite multiplicities, and all eigenvalues of $L(\cdot, \alpha), \alpha \geq 0$, lie in the closed upper half-plane and on the imaginary axis and are symmetric with respect to the imaginary axis.

Proof. As in [2, Section 3], we can argue that for all $\lambda \in$ $\mathbb{C}, L(\lambda, \alpha)$ is a relatively compact perturbation of $L(0,0)$, where $L(0,0)$ is well known to be a Fredholm operator. The statement on the location of the spectrum follows as in [2, Lemma 3.1].

\section{Asymptotics of Eigenvalues for $g=0$}

In this section, we investigate the boundary value problem (1), (2) with $g=0$. We count all eigenvalues with their proper multiplicities and develop a formula for the asymptotic distribution of the eigenvalues, which we will use to obtain the corresponding formula for general $g$. Observe that for 
$g=0$, the quasi-derivatives $y^{[j]}$ coincide with the standard derivatives $y^{(j)}$. We take the canonical fundamental system $y_{j}(\cdot, \lambda), j=1, \ldots, 4$, of (1) with $y_{j}^{(m)}(0)=\delta_{j, m+1}$ for $m=$ $0, \ldots, 3$. It is well known that the functions $y_{j}(\cdot, \lambda)$ are analytic on $\mathbb{C}$ with respect to $\lambda$. Putting

$$
M(\lambda)=\left(B_{i}(\lambda) y_{j}(\cdot, \lambda)\right)_{i, j=1}^{4},
$$

the eigenvalues of the boundary value problem (1), (2) are the eigenvalues of the analytic matrix function $M$, where the corresponding geometric and algebraic multiplicities coincide, see [10, Theorem 6.3.2].

Setting $\lambda=\mu^{2}$ and

$$
y(x, \mu)=\frac{1}{2 \mu^{3}} \sinh (\mu x)-\frac{1}{2 \mu^{3}} \sin (\mu x),
$$

it is easy to see that

$$
y_{j}(x, \lambda)=y^{(4-j)}(x, \mu), \quad j=1, \ldots, 4 .
$$

The first and the second rows of $M(\lambda)$ have exactly two nonzero entries (for $\lambda \neq 0$ ), and these nonzero entries are: $B_{1}(\lambda) y_{2}(\cdot, \lambda)=-i \alpha \mu^{2}$ and $B_{1}(\lambda) y_{3}(\cdot, \lambda)=1$ while $B_{2}(\lambda) y_{1}(\cdot, \lambda)=i \alpha \mu^{2}$ and $B_{2}(\lambda) y_{4}(\cdot, \lambda)=1$. Therefore,

$M(\lambda)$

$$
=\left(\begin{array}{cccc}
0 & -i \alpha \mu^{2} & 1 & 0 \\
i \alpha \mu^{2} & 0 & 0 & 1 \\
B_{3}\left(\mu^{2}\right) y_{1} & B_{3}\left(\mu^{2}\right) y_{2} & B_{3}\left(\mu^{2}\right) y_{3} & B_{3}\left(\mu^{2}\right) y_{4} \\
B_{4}\left(\mu^{2}\right) y_{1} & B_{4}\left(\mu^{2}\right) y_{2} & B_{4}\left(\mu^{2}\right) y_{3} & B_{4}\left(\mu^{2}\right) y_{4}
\end{array}\right) .
$$

An expansion of $\operatorname{det} M(\lambda)=\phi(\mu)$ gives

$$
\phi(\mu)=-\alpha^{2} \mu^{4} \Phi_{1}(\mu)+i \alpha \mu^{2} \Phi_{2}(\mu)+i \alpha \mu^{2} \Phi_{3}(\mu)+\Phi_{4}(\mu),
$$

where

$$
\Phi_{j}(\mu)=\operatorname{det}\left(\begin{array}{ll}
B_{3}\left(\mu^{2}\right) y_{\sigma_{j, 1}}(\cdot, \mu) & B_{3}\left(\mu^{2}\right) y_{\sigma_{j, 2}}(\cdot, \mu) \\
B_{4}\left(\mu^{2}\right) y_{\sigma_{j, 1}}(\cdot, \mu) & B_{4}\left(\mu^{2}\right) y_{\sigma_{j, 2}}(\cdot, \mu)
\end{array}\right),
$$

with

$$
\left(\sigma_{j, 1}, \sigma_{j, 2}\right)= \begin{cases}(3,4) & \text { if } j=1 \\ (1,3) & \text { if } j=2 \\ (2,4) & \text { if } j=3 \\ (1,2) & \text { if } j=4\end{cases}
$$

In view of (11), (12), we get that

$$
\begin{gathered}
\Phi_{j}(\mu) \\
=\left[i \alpha \mu ^ { 2 } \left\{y_{\sigma_{j, 1}}^{\prime}(a, \mu) y_{\sigma_{j, 2}}^{(3)}(a, \mu)\right.\right. \\
-y_{\sigma_{j, 2}}^{\prime}(a, \mu) y_{\sigma_{j, 1}}^{(3)}(a, \mu) \\
+y_{\sigma_{j, 2}}^{\prime \prime}(a, \mu) y_{\sigma_{j, 1}}(a, \mu) \\
\left.-y_{\sigma_{j, 1}}^{\prime \prime}(a, \mu) y_{\sigma_{j, 2}}(a, \mu)\right\} \\
+\alpha^{2} \mu^{4}\left\{y_{\sigma_{j, 1}}^{\prime}(a, \mu) y_{\sigma_{j, 2}}(a, \mu)\right. \\
\left.-y_{\sigma_{j, 2}}^{\prime}(a, \mu) y_{\sigma_{j, 1}}(a, \mu)\right\} \\
+y_{\sigma_{j, 1}}^{\prime \prime}(a, \mu) y_{\sigma_{j, 2}}^{(3)}(a, \mu) \\
\left.-y_{\sigma_{j, 2}}^{\prime \prime}(a, \mu) y_{\sigma_{j, 1}}^{(3)}(a, \mu)\right] .
\end{gathered}
$$

Observing that the $y_{j}$ are given by (14) and (15) a straightforward calculation leads to

$$
\begin{aligned}
\Phi_{1}(\mu)= & \frac{1}{2} i \alpha \mu(\sin (\mu a) \cosh (\mu a)+\cos (\mu a) \sinh (\mu a)) \\
& +\frac{1}{2 \mu} i \alpha(\sin (\mu a) \cosh (\mu a) \\
& \quad-\cos (\mu a) \sinh (\mu a)) \\
& -\frac{1}{2} \alpha^{2}(1-\cos (\mu a) \cosh (\mu a)) \\
& +\frac{1}{2}(1+\cos (\mu a) \cosh (\mu a)), \\
\Phi_{2}(\mu)= & -i \alpha \mu^{4} \sin (\mu a) \sinh (\mu a) \\
& +i \alpha \mu^{2} \cos (\mu a) \cosh (\mu a)-\frac{1}{2}\left(1+\alpha^{2}\right) \\
& \times \mu^{3}(\sin (\mu a) \cosh (\mu a)+\cos (\mu a) \sinh (\mu a)), \\
\Phi_{3}(\mu)= & i \alpha \mu^{2} \cos (\mu a) \cosh (\mu a)+i \alpha \sin (\mu a) \sinh (\mu a) \\
& -\frac{1}{2}\left(1+\alpha^{2}\right) \\
& \times \mu(\sin (\mu a) \cosh (\mu a)-\cos (\mu a) \sinh (\mu a)), \\
\Phi_{4}(\mu)= & -\frac{1}{2} i \alpha \mu^{5}(\sin (\mu a) \cosh (\mu a)+\cos (\mu a) \sinh (\mu a)) \\
& -\frac{1}{2} i \alpha \mu^{3}(\sin (\mu a) \cosh (\mu a)-\cos (\mu a) \sinh (\mu a)) \\
& -\frac{1}{2} \alpha^{2} \mu^{4}(1+\cos (\mu a) \cosh (\mu a)) \\
+ & \frac{1}{2} \mu^{4}(1-\cos (\mu a) \cosh (\mu a)) . \\
&
\end{aligned}
$$


It follows that the term with the highest $\mu$-power in $\phi$ comes from $\Phi_{2}$ and is a nonzero multiple of

$$
\phi_{0}(\mu):=\mu^{6} \sin (\mu a) \sinh (\mu a)
$$

The following result on the zeros of $\phi_{0}$, with proper counting, is obvious.

Lemma 3. $\phi_{0}$ has a zero of multiplicity 8 at 0 , simple zeros at

$$
\tilde{\mu}_{k}=(k-2) \frac{\pi}{a}, \quad k=3,4, \ldots,
$$

simple zeros at $-\widetilde{\mu}_{k}, \widetilde{\mu}_{-k}=i \widetilde{\mu}_{k}$ and $-i \widetilde{\mu}_{k}$ for $k=3,4 \ldots$, and no other zeros.

Proposition 4. For $g=0$, there exists a positive integer $k_{0}$ such that the eigenvalues $\widehat{\lambda}_{k}, k \in \mathbb{Z} \backslash\{0\}$, counted with multiplicity, of the problem (1), (9)-(12) can be enumerated in such a way that the eigenvalues $\hat{\lambda}_{k}$ are pure imaginary for $|k|<k_{0}$, and $\hat{\lambda}_{-k}=-\widehat{\hat{\lambda}}_{k}$ for $k \geq k_{0}$. For $k>0$, we can write that $\hat{\lambda}_{k}=\widehat{\mu}_{k}^{2}$, where the $\widehat{\mu}_{k}$ have the following asymptotic representation as $k \rightarrow \infty$ :

$$
\widehat{\mu}_{k}=(k-2) \frac{\pi}{a}+o(1) .
$$

In particular, the number of pure imaginary eigenvalues is even.

Proof. It follows from (17) and (21) that

$$
\begin{aligned}
\phi(\mu)= & \alpha^{2} \mu^{6} \sin (\mu a) \sinh (\mu a)-i\left(\alpha^{3}+\alpha\right) \\
& \times \mu^{5}(\sin (\mu a) \cosh (\mu a)+\cos (\mu a) \sinh (\mu a)) \\
& -\alpha^{2} \mu^{4}(1+3 \cos (\mu a) \cosh (\mu a)) \\
& -\alpha^{2} \mu^{2} \sin (\mu a) \sinh (\mu a) \\
& +\frac{1}{2} \alpha^{4} \mu^{4}(1-\cos (\mu a) \cosh (\mu a)) \\
& +\frac{1}{2} \mu^{4}(1-\cos (\mu a) \cosh (\mu a))-i\left(\alpha^{3}+\alpha\right) \\
& \times \mu^{3}(\sin (\mu a) \cosh (\mu a)-\cos (\mu a) \sinh (\mu a)) .
\end{aligned}
$$

Up to the constant factor $\alpha^{2}$, the first term equals $\phi_{0}(\mu)$. Considering that, therefore,

$$
\phi_{1}(\mu):=\frac{\phi(\mu)-\alpha^{2} \phi_{0}(\mu)}{\phi_{0}(\mu)}
$$

for $\phi_{0}(\mu) \neq 0$, that is, $\sin (\mu a) \neq 0$ and $\sinh (\mu a) \neq 0$. It follows that

$$
\begin{aligned}
\phi_{1}(\mu)= & -\frac{i\left(\alpha^{3}+\alpha\right)}{\mu}(\cot (\mu a)+\operatorname{coth}(\mu a)) \\
& -\frac{\alpha^{2}}{\mu^{2}}\left[\frac{1}{\sin (\mu a) \sinh (\mu a)}+3 \cot (\mu a) \operatorname{coth}(\mu a)\right] \\
& -\frac{\alpha^{2}}{\mu^{4}} \\
& +\frac{\alpha^{4}}{2 \mu^{2}}\left[\frac{1}{\sin (\mu a) \sinh (\mu a)}-\cot (\mu a) \operatorname{coth}(\mu a)\right] \\
& +\frac{1}{2 \mu^{2}}\left[\frac{1}{\sin (\mu a) \sinh (\mu a)}-\cot (\mu a) \operatorname{coth}(\mu a)\right] \\
& -\frac{i\left(\alpha^{3}+\alpha\right)}{\mu^{3}}(\cot (\mu a)-\operatorname{coth}(\mu a)) .
\end{aligned}
$$

Fix $\varepsilon \in(0, \pi / 2 a)$, and for $k=3,4, \ldots$, let $R_{k, \varepsilon}$ be the squares determined by the vertices $(k-2)(\pi / a) \pm \varepsilon \pm i \varepsilon$, $k=3,4, \ldots$. These squares do not intersect due to $\varepsilon<\pi / 2 a$. There exists $C_{1}(\varepsilon)>0$ such that $|\operatorname{coth}(\mu a)|<C_{1}(\varepsilon)$ for all $\mu$ on the squares $R_{k, \varepsilon}, k \geq 3$. By periodicity, there are numbers $C_{2}(\varepsilon)$ and $C_{3}(\varepsilon)$ such that $|\sin (\mu a)|>C_{2}(\varepsilon)$ and $|\cot (\mu a)|<C_{3}(\varepsilon)$ for all $\mu$ on the boundary of the squares $R_{k, \varepsilon}$ for all $k \geq 3$. Since $|\sinh (\mu a)| \geq|\sinh ((\operatorname{Re} \mu) a)|$, it follows that there is $k_{1}(\varepsilon) \in \mathbb{N}$ such that for all $\mu$ on the squares $R_{k, \varepsilon}$, where $k>k_{1}(\varepsilon)$, the estimate $\left|\phi_{1}(\mu)\right|<1$ holds. By Lemma 3, $\phi_{0}$ has exactly one simple zero inside $R_{k, \varepsilon}$. Hence, it follows from Rouchés theorem that there is exactly one (simple) zero $\widehat{\mu}_{k}$ of $\phi$ in each $R_{k, \varepsilon}$ for $k \geq k_{1}(\varepsilon)$, which proves the existence of zeros $\widehat{\mu}_{k}$ of $\phi$ with $\widehat{\mu}_{k}=\widetilde{\mu}_{k}+o(1)$ as $k \rightarrow \infty$.

Let

$$
\begin{aligned}
\widetilde{\phi}_{0}(\mu)= & \alpha^{2} \mu^{6} \sin (\mu a) \sinh (\mu a) \\
& -\alpha^{2} \mu^{4}(1+3 \cos (\mu a) \cosh (\mu a)) \\
& -\alpha^{2} \mu^{2} \sin (\mu a) \sinh (\mu a) \\
& +\frac{1}{2} \alpha^{4} \mu^{4}(1-\cos (\mu a) \cosh (\mu a)) \\
& +\frac{1}{2} \mu^{4}(1-\cos (\mu a) \cosh (\mu a)), \\
\widetilde{\phi}_{1}(\mu)= & -i\left(\alpha^{3}+\alpha\right) \\
& \times \mu^{5}(\sin (\mu a) \cosh (\mu a)+\cos (\mu a) \sinh (\mu a)) \\
- & i\left(\alpha^{3}+\alpha\right) \\
& \times \mu^{3}(\sin (\mu a) \cosh (\mu a)-\cos (\mu a) \sinh (\mu a)) .
\end{aligned}
$$


Then, $\phi(\mu)=\widetilde{\phi}_{0}(\mu)+\widetilde{\phi}_{1}(\mu)$, and for all $\mu \in \mathbb{C}$,

$$
\phi(i \mu)=\widetilde{\phi}_{0}(\mu)-\widetilde{\phi}_{1}(\mu)=\overline{\phi(\bar{\mu})}
$$

Observing that $\phi$ is an even function, it follows altogether that $\phi$ has zeros $\pm \widehat{\mu}_{k}, \pm \widehat{\mu}_{-k}$ for $k>k_{1}(\varepsilon)$ with $\widehat{\mu}_{-k}=i \overline{\widehat{\mu}}_{k}$.

We still have to show that the stated counting and asymptotic behaviour describes all zeros of $\phi$. To this end, we are going to estimate $\phi_{1}$ on the squares $S_{k}, k \in \mathbb{N}$, whose vertices are $\pm(k+(1 / 2))(\pi / a) \pm i(k+(1 / 2))(\pi / a)$. For $\mu=$ $x+i y, x, y \in \mathbb{R}$ and $x \neq 0$, we have

$$
\operatorname{coth}(\mu a)=\frac{e^{(a x+i a y)}+e^{-(a x+i a y)}}{e^{(a x+i a y)}-e^{-(a x+i a y)}} \longrightarrow \pm 1
$$

uniformly in $y$ as $x \rightarrow \pm \infty$. Hence, there are $\xi \geq 1$ and $\widetilde{k}_{0}>0$ such that for all $k \in \mathbb{N}, k \geq \widetilde{k}_{0}$ and $\gamma \in \mathbb{R}$,

$$
\left|\operatorname{coth}\left(\left(\frac{(k+(1 / 2)) \pi}{a}+i \gamma\right) a\right)\right| \leq \xi .
$$

Note that for $k \in \mathbb{N}$ and $\gamma \in \mathbb{R}$,

$$
\cot \left(\left(\frac{(k+(1 / 2)) \pi}{a}+i \gamma\right) a\right)=-\tan (i \gamma a)=-i \tanh (\gamma a) .
$$

Hence, we have shown that for $k \in \mathbb{N}$ and $\gamma \in \mathbb{R}$,

$$
\left|\cot \left(\left(\frac{(k+(1 / 2)) \pi}{a}+i \gamma\right) a\right)\right| \leq 1 \text {. }
$$

Furthermore, we will make use of the estimates

$$
\begin{gathered}
\left|\sinh \left(\left(\frac{(k+(1 / 2)) \pi}{a}+i \gamma\right) a\right)\right| \geq\left|\sinh \left(\left(k+\frac{1}{2}\right) \pi\right)\right|, \\
\left|\sin \left(\left(\frac{(k+(1 / 2)) \pi}{a}+i \gamma\right) a\right)\right|=\cosh (\gamma a) \geq 1,
\end{gathered}
$$

which hold for all $k \in \mathbb{Z}$ and all $\gamma \in \mathbb{R}$. Therefore, it follows from (31), (33)-(34), and the symmetry of $\phi$, see (29), that there is $\widetilde{k}_{1} \geq \widetilde{k}_{0}$ such that $\left|\phi_{1}(\mu)\right|<1$ for all $\mu \in S_{k}$ with $k>\widetilde{k}_{1}$. Again from the definition of $\phi_{1}$ in (27) and Rouché's theorem, we conclude that the functions $\phi_{0}$ and $\phi$ have the same number of zeros in the square $S_{k}$, for $k \in \mathbb{N}$ with $k \geq \widetilde{k}_{1}$. Since $\phi_{0}$ has $4 k+8$ zeros inside $S_{k}$ and thus $4 k+8+4$ zeros inside $S_{k+1}$, it follows that all such zeros of $\phi$ are accounted for by $\pm \widehat{\mu}_{j}$ for $0<|j| \leq k+2$ and sufficiently large $k$.

Finally, $\widehat{\lambda}_{k}=\widehat{\mu}_{k}^{2}$ account for all eigenvalues of the problem (1)-(2) since each of these eigenvalues gives rise to two zeros of $\phi$, counted with multiplicity. By Proposition 2, all eigenvalues with nonzero real part occur in pairs $\hat{\lambda}_{k},-\overline{\hat{\lambda}}_{k}$, which shows that we can index all such eigenvalues as $\hat{\lambda}_{-k}=-\overline{\hat{\lambda}}_{k}$. Since there is an even number of remaining indices, the number of pure imaginary eigenvalues must be even.

\section{Birkhoff Regularity}

We refer to [10, Definition 7.3.1] for the definition of Birkhoff regularity.

Proposition 5. The boundary value problem (1), (9)-(12) is Birkhoff regular for $\alpha>0$ with respect to the eigenvalue parameter $\mu$ given by $\lambda=\mu^{2}$.

Proof. The characteristic function of (1) as defined in [10, (7.1.4)] is $\pi(\rho)=\rho^{4}-1$, and its zeros are $i^{k-1}, k=1, \ldots, 4$. We can choose

$$
C(x, \mu)=\operatorname{diag}\left(1, \mu, \mu^{2}, \mu^{3}\right)\left(i^{(k-1)(j-1)}\right)_{k, j=1}^{4}
$$

according to [10, Theorem 7.2.4.A]. The boundary condition (9)-(12) can be written in the form

$$
\begin{array}{r}
B_{j}(\lambda) y=\widehat{B}_{j}(\mu)\left(y\left(a_{j}\right), y^{\prime}\left(a_{j}\right), y^{\prime \prime}\left(a_{j}\right), y^{(3)}\left(a_{j}\right)\right), \\
j=1,2,3,4 .
\end{array}
$$

Thus, the boundary matrices defined in $[10,(7.3 .1)]$ are given by

$$
\begin{aligned}
& W^{(0)}(\mu)=\left(\begin{array}{c}
\widehat{B}_{1}(\mu) \\
\widehat{B}_{2}(\mu) \\
0 \\
0
\end{array}\right) C(0, \mu), \\
& W^{(1)}(\mu)=\left(\begin{array}{c}
0 \\
0 \\
\widehat{B}_{3}(\mu) \\
\widehat{B}_{4}(\mu)
\end{array}\right) C(a, \mu),
\end{aligned}
$$

where

$$
\begin{gathered}
\widehat{B}_{1}(\mu)=\left(0,-i \alpha \mu^{2}, 1,0\right), \\
\widehat{B}_{2}(\mu)=\left(i \alpha \mu^{2},-g(0), 0,1\right), \\
\widehat{B}_{3}(\mu)=\left(0, i \alpha \mu^{2}, 1,0\right), \\
\widehat{B}_{4}(\mu)=\left(-i \alpha \mu^{2},-g(a), 0,1\right) .
\end{gathered}
$$

Choosing $C_{2}(\mu)=\operatorname{diag}\left(\mu^{3}, \mu^{3}, \mu^{3}, \mu^{3}\right)$, it follows that $C_{2}(\mu)^{-1} W^{(j)}(\mu)=W_{0}^{(j)}+O\left(\mu^{-1}\right)$, where

$$
\begin{aligned}
& W_{0}^{(0)}=\left(\begin{array}{cccc}
-i \alpha & \alpha & i \alpha & -\alpha \\
1 & -i & -1 & i \\
0 & 0 & 0 & 0 \\
0 & 0 & 0 & 0
\end{array}\right), \\
& W_{0}^{(1)}=\left(\begin{array}{cccc}
0 & 0 & 0 & 0 \\
0 & 0 & 0 & 0 \\
i \alpha & -\alpha & -i \alpha & \alpha \\
1 & -i & -1 & i
\end{array}\right) .
\end{aligned}
$$


The Birkhoff matrices are

$$
W_{0}^{(0)} \Delta_{j}+W_{0}^{(1)}\left(I-\Delta_{j}\right)
$$

where $\Delta_{j}, j=1,2,3,4$ are the $4 \times 4$ diagonal matrices with 2 consecutive ones and 2 consecutive zeros in the diagonal in a cyclic arrangement, see [10, Definition 7.3.1 and Proposition 4.1.7]. It is easy to see that after a permutation of columns, the matrices (40) are block diagonal matrices consisting of $2 \times 2$ blocks taken from two consecutive columns (in the sense of cyclic arrangement) of the first two rows of $W_{0}^{(0)}$ and the last two rows of $W_{0}^{(1)}$, respectively. Hence, the determinants of the Birkhoff matrices (40) are

$$
\begin{aligned}
(-1)^{j-1} & \left|\begin{array}{cc}
-i^{j} \alpha & -i^{j+1} \alpha \\
(-i)^{j-1} & (-i)^{j}
\end{array}\right|\left|\begin{array}{cc}
i^{j+2} \alpha & i^{j+3} \alpha \\
(-i)^{j+1} & (-i)^{j+2}
\end{array}\right| \\
= & (-1)^{j-1}(-2 \alpha)(2 \alpha) \\
= & (-1)^{j} 4 \alpha^{2} \neq 0 .
\end{aligned}
$$

Thus, the problem (1), (9)-(12) is Birkhoff regular for $\alpha>0$.

\section{Asymptotic Expansions of Eigenvalues}

With $\lambda=\mu^{2}, D(\mu)=\operatorname{det}\left(B_{i}\left(\mu^{2}\right) y_{j}(\cdot, \mu)\right)_{i, j=1}^{4}$ defines a characteristic determinant of the problem (1), (9)-(12) with respect to the fundamental system $y_{j}, j=1,2,3,4$ considered in Section 3. Observe that $\phi$ is the corresponding characteristic determinant for $g=0$. Due to the Birkhoff regularity, $g$ only influences lower order terms in $D$. Together with the estimates in Section 3, it can be inferred that outside the interior of the small squares $R_{k, \varepsilon},-R_{k, \varepsilon}, i R_{k, \varepsilon}$, $-i R_{k, \varepsilon}$ around the zeros of $\phi_{0},\left|D(\mu)-\phi_{0}(\mu)\right|<\left|\phi_{0}(\mu)\right|$ if $|\mu|$ is sufficiently large. Since the fundamental system $y_{j}$, $j=1,2,3,4$, depends analytically on $\mu$, also $D$ depends analytically on $\mu$. Hence, applying Rouchés theorem both to the large squares $S_{k}$ and to the small squares which are sufficiently far away from the origin, it follows that the eigenvalues of the boundary value problem for general $g$ have the same asymptotic distribution as for $g=0$. Hence Proposition 4 leads to the following.

Proposition 6. For $g \in C^{1}[0, a]$, there exists a positive integer $k_{0}$ such that the eigenvalues $\lambda_{k}$, counted with multiplicity, of the problem (1), (9)-(12), where $k \in \mathbb{Z} \backslash\{0\}$ can be enumerated in such a way that the eigenvalues $\lambda_{k}$ are pure imaginary for $|k|<$ $k_{0}$, and $\lambda_{-k}=-\overline{\lambda_{k}}$ for $k \geq k_{0}$. For $k>0$, we can write $\lambda_{k}=\mu_{k}^{2}$, where the $\mu_{k}$ have the following asymptotic representation as $k \rightarrow \infty$ :

$$
\mu_{k}=(k-2) \frac{\pi}{a}+o(1) .
$$

In particular, the number of pure imaginary eigenvalues is even.

In the remainder of the section, we establish more precise asymptotic expansions of the eigenvalues. For this, it is more convenient to replace $\left(y_{j}\right)_{j=1}^{4}$ with the asymptotic fundamental system $\left(\eta_{\nu}\right)_{v=1}^{4}$ obtained in [10, Theorem 8.2.1], (1), which can be written as

$$
\eta_{\nu}^{(j)}(x, \mu)=\delta_{\nu, j}(x, \mu) e^{i^{\nu-1} \mu x} ; \quad v=1, \ldots, 4 ; j=0, \ldots, 3,
$$

where

$$
\begin{aligned}
\delta_{\nu, j}(x, \mu)= & {\left[\frac{d^{j}}{d x^{j}}\right]\left\{\sum_{r=0}^{4}\left(\mu i^{\nu-1}\right)^{-r} \varphi_{r}(x) e^{i^{\nu-1} \mu x}\right\} e^{-i^{\nu-1} \mu x} } \\
& +o\left(\mu^{-4+j}\right),
\end{aligned}
$$

and $\left[d^{j} / d x^{j}\right]$ means that we omit those terms of the Leibniz expansion which contain a function $\varphi_{r}^{(k)}$ with $k>4-r$. Since the coefficient of $y^{(3)}$ in (1) is zero, we have $\varphi_{0}(x)=1$, see [10, (8.2.3)].

We will now determine the functions $\varphi_{1}$ and $\varphi_{2}$. In this regard, observe that $n_{0}=0$ and $l=4$ in the notation of $[10,(8.1 .2)$ and $(8.1 .3)]$, see $[10$, Theorem 8.1.2]. From [10, (8.2.45)], we know that

$$
\varphi_{r}=\varphi_{1, r}=\varepsilon_{1}^{\top} V Q^{[r]} \varepsilon_{1},
$$

where $\varepsilon_{v}$ is the $v$ th unit vector in $\mathbb{C}^{4}, V=\left(i^{(j-1)(k-1)}\right)_{j, k=1}^{4}$, and $Q^{[r]}$ are $4 \times 4$ matrices given by $[10,(8.2 .28),(8.2 .33)$, and (8.2.34)], that is, $Q^{[0]}=I_{4}$,

$$
\begin{gathered}
\Omega_{4} Q^{[1]}-Q^{[1]} \Omega_{4}=Q^{[0]^{\prime}}=0, \\
\Omega_{4} Q^{[2]}-Q^{[2]} \Omega_{4}=Q^{[1]^{\prime}}-\frac{1}{4} g \Omega_{4} \varepsilon \varepsilon^{\top} \Omega_{4}^{-2} Q^{[0]}, \\
0=\varepsilon_{v}^{\top}\left(Q^{[2]^{\prime}}+\frac{1}{4} \sum_{j=1}^{2} k_{3-j} \Omega_{4} \varepsilon \varepsilon^{\top} \Omega_{4}^{-1-j} Q^{[2-j]}\right) \varepsilon_{v} \\
(\nu=1,2,3,4),
\end{gathered}
$$

where $k_{2}=-g, k_{1}=-g^{\prime}, \Omega_{4}=\operatorname{diag}(1, i,-1,-i)$ and $\varepsilon^{\top}=(1,1,1,1)$. Letting $G(x)=\int_{0}^{x} g(t) d t$, a lengthy but straightforward calculation gives

$$
\varphi_{1}=\frac{1}{4} G, \quad \varphi_{2}=\frac{1}{32} G^{2}-\frac{1}{8} g,
$$

and thus

$$
\begin{aligned}
\eta_{\nu}(x, \mu)=[( & +\frac{1}{4} i^{-\nu+1} G(x) \mu^{-1} \\
& \left.+(-1)^{\nu-1}\left(\frac{1}{32} G(x)^{2}-\frac{1}{8} g(x)\right) \mu^{-2}\right) \\
& \left.+\left\{o\left(\mu^{-2}\right)\right\}_{\infty}\right] e^{i^{\nu-1} \mu x}
\end{aligned}
$$

for $v=1, \ldots, 4$, where $\{o(\cdot)\}_{\infty}$ means that the estimate is uniform in $x$. 
In view of (44), the characteristic determinant of (1), (9)(12) with respect to the fundamental system $\left(\eta_{k}\right)_{k=1}^{4}$ is

$$
\widetilde{D}(\mu)=\operatorname{det}\left(B_{j}\left(\mu^{2}\right) \eta_{k}(\cdot, \mu)\right)_{j, k=1}^{4}=\operatorname{det}\left(\gamma_{j k} \exp \left(\varepsilon_{j k}\right)\right)_{j, k=1}^{4}
$$

where

$$
\begin{gathered}
\varepsilon_{1 k}=\varepsilon_{2 k}=0, \quad \varepsilon_{3 k}=\varepsilon_{4 k}=i^{k-1} \mu a, \\
\gamma_{1 k}=\delta_{k, 2}(0, \mu)-i \alpha \mu^{2} \delta_{k, 1}(0, \mu), \\
\gamma_{2 k}=\delta_{k, 3}(0, \mu)-g(0) \delta_{k, 1}(0, \mu)+i \alpha \mu^{2} \delta_{k, 0}(0, \mu), \\
\gamma_{3 k}=\delta_{k, 2}(a, \mu)+i \alpha \mu^{2} \delta_{k, 1}(a, \mu), \\
\gamma_{4 k}=\delta_{k, 3}(a, \mu)-g(a) \delta_{k, 1}(a, \mu)-i \alpha \mu^{2} \delta_{k, 0}(a, \mu) .
\end{gathered}
$$

An expansion of $\widetilde{D}$ leads to

$$
\widetilde{D}(\mu)=\sum_{m=1}^{5} \psi_{m}(\mu) e^{\omega_{m} \mu a}
$$

where $\omega_{1}=1+i, \omega_{2}=-1+i, \omega_{3}=-1-i, \omega_{4}=1-i$, $\omega_{5}=0$, and each of the functions $\psi_{1}, \ldots, \psi_{5}$ has asymptotic representations of the form $c_{k} \mu^{k}+c_{k-1} \mu^{k-1}+\cdots+c_{k_{0}} \mu^{k_{0}}+o\left(\mu^{k_{0}}\right)$.

It follows from (51) that

$$
D_{1}(\mu):=\widetilde{D}(\mu) e^{-\omega_{1} \mu a}=\psi_{1}(\mu)+\sum_{m=2}^{5} \psi_{m}(\mu) e^{\left(\omega_{m}-\omega_{1}\right) \mu a}
$$

where $\omega_{2}-\omega_{1}=-2, \omega_{3}-\omega_{1}=-2-2 i, \omega_{4}-\omega_{1}=-2 i, \omega_{5}-$ $\omega_{1}=-1-i$. If $\arg \mu \in[-(3 \pi / 8), \pi / 8]$, we have $\left|e^{\left(\omega_{m}-\omega_{1}\right) \mu a}\right| \leq$ $e^{-\sin (\pi / 8)|\mu| a}$ for $m=2,3,5$ and the terms $\psi_{m}(\mu) e^{\left(\omega_{m}-\omega_{1}\right) \mu a}$ for $m=2,3,5$, can be absorbed by $\psi_{1}(\mu)$ as they are of the form $o\left(\mu^{-s}\right)$ for any integer $s$. Hence, for $\arg \mu \in[-(3 \pi / 8), \pi / 8]$,

$$
D_{1}(\mu)=\psi_{1}(\mu)+\psi_{4}(\mu) e^{\left(\omega_{4}-\omega_{1}\right) \mu a}=\psi_{1}(\mu)+\psi_{4}(\mu) e^{-2 i \mu a},
$$

where

$$
\begin{aligned}
& \psi_{1}(\mu)=\left[\gamma_{13} \gamma_{24}-\gamma_{14} \gamma_{23}\right]\left[\gamma_{31} \gamma_{42}-\gamma_{32} \gamma_{41}\right] \\
& \psi_{4}(\mu)=\left[\gamma_{12} \gamma_{23}-\gamma_{13} \gamma_{22}\right]\left[\gamma_{31} \gamma_{44}-\gamma_{34} \gamma_{41}\right]
\end{aligned}
$$

A straightforward calculation gives that

$$
\begin{aligned}
& \gamma_{13} \gamma_{24}-\gamma_{14} \gamma_{23} \\
&=-2 \alpha \mu^{6}-\left(1+\alpha^{2}\right)(1-i) \mu^{5}+2 i \alpha \mu^{4}+o\left(\mu^{4}\right), \\
& \gamma_{31} \gamma_{42}-\gamma_{32} \gamma_{41} \\
&=2 \alpha \mu^{6}+(1-i) \mu^{5}\left(1+\alpha^{2}+2 \alpha \varphi_{1}(a)\right) \\
& \quad-2 i \mu^{4}\left(\alpha\left(1+\varphi_{1}(a)^{2}\right)+\left(1+\alpha^{2}\right) \varphi_{1}(a)\right)+o\left(\mu^{4}\right), \\
& \gamma_{12} \gamma_{23}-\gamma_{13} \gamma_{22} \\
&=-2 \alpha \mu^{6}+\left(1+\alpha^{2}\right)(1+i) \mu^{5}-2 i \alpha \mu^{4}+o\left(\mu^{4}\right), \\
& \gamma_{31} \gamma_{44}-\gamma_{34} \gamma_{41} \\
&=-2 \alpha \mu^{6}+(1+i) \mu^{5}\left(1+\alpha^{2}-2 \alpha \varphi_{1}(a)\right) \\
& \quad-2 i \mu^{4}\left(\alpha\left(1+\varphi_{1}(a)^{2}\right)-\left(1+\alpha^{2}\right) \varphi_{1}(a)\right)+o\left(\mu^{4}\right) .
\end{aligned}
$$

Hence,

$$
\begin{aligned}
\mu^{-12} \psi_{1}(\mu)= & -4 \alpha^{2}-(1-i) \alpha\left[4\left(1+\alpha^{2}\right)+\alpha G(a)\right] \mu^{-1} \\
& +i\left[\frac{1}{4} \alpha^{2} G^{2}(a)+2 \alpha\left(1+\alpha^{2}\right) G(a)\right. \\
& \left.+2\left(1+\alpha^{2}\right)^{2}+8 \alpha^{2}\right] \mu^{-2}+o\left(\mu^{-2}\right) \\
\mu^{-12} \psi_{4}(\mu)= & 4 \alpha^{2}-(1+i) \alpha\left[4\left(1+\alpha^{2}\right)-\alpha G(a)\right] \mu^{-1} \\
+ & i\left[\frac{1}{4} \alpha^{2} G^{2}(a)-2 \alpha\left(1+\alpha^{2}\right) G(a)\right. \\
& \left.+2\left(1+\alpha^{2}\right)^{2}+8 \alpha^{2}\right] \mu^{-2}+o\left(\mu^{-2}\right) .
\end{aligned}
$$

We know by Proposition 6 that the zeros $\mu_{k}$ of $\widetilde{D}$ satisfy the asymptotic representations $\mu_{k}=k(\pi / a)+\tau_{0}+o(1)$ as $k \rightarrow$ $\infty$. In order to improve on these asymptotic representations, write

$$
\begin{array}{r}
\mu_{k}=k \frac{\pi}{a}+\tau(k), \quad \tau(k)=\sum_{m=0}^{2} \tau_{m} k^{-m}+o\left(k^{-2}\right), \\
k=1,2, \ldots .
\end{array}
$$

Because of the symmetry of the eigenvalues, we will only need to find the asymptotic expansions as $k \rightarrow \infty$. We know from Proposition 6 that $\tau_{0}=-(2 \pi / k)$, and it is our aim to find $\tau_{1}$ and $\tau_{2}$. To this end, we will substitute (58) into $D_{1}\left(\mu_{k}\right)=0$, and we will then compare the coefficients of $k^{0}, k^{-1}$, and $k^{-2}$. 
Observe that

$$
\begin{aligned}
e^{-2 i \mu_{k} a}= & e^{-2 i \tau(k) a}=e^{-2 i \tau_{0} a} \exp \left(-2 i a\left(\frac{\tau_{1}}{k}+\frac{\tau_{2}}{k^{2}}+o\left(k^{-2}\right)\right)\right) \\
= & e^{-2 i \tau_{0} a} \\
& \times\left(1-2 i a \tau_{1} \frac{1}{k}-\left(2 a^{2} \tau_{1}^{2}+2 i a \tau_{2}\right) \frac{1}{k^{2}}+o\left(k^{-2}\right)\right),
\end{aligned}
$$

while

$$
\frac{1}{\mu_{k}}=\frac{a}{\pi k}\left(1+\frac{a \tau(k)}{k \pi}\right)^{-1}=\frac{a}{k \pi}-\frac{a^{2} \tau_{0}}{k^{2} \pi^{2}}+o\left(k^{-2}\right) .
$$

Using (53), $D_{1}\left(\mu_{k}\right)=0$ can be written as

$$
\mu_{k}^{-12} \psi_{1}\left(\mu_{k}\right)+\mu_{k}^{-12} \psi_{4}\left(\mu_{k}\right) e^{-2 i \mu_{k} a}=0 .
$$

Substituting (56), (57), (59), and (60) into (61) and comparing the coefficients of $k^{0}, k^{-1}$, and $k^{-2}$, we get

Theorem 7. For $g \in C^{1}[0, a]$, there exists a positive integer $k_{0}$ such that the eigenvalues $\lambda_{k}, k \in \mathbb{Z}$, counted with multiplicity, of the problem (1), (9)-(12), where $k \in \mathbb{Z} \backslash\{0\}$ can be enumerated in such a way that the eigenvalues $\lambda_{k}$ are pure imaginary for $|k|<k_{0}$, and $\lambda_{-k}=-\overline{\lambda_{k}}$ for $k \geq k_{0}$, where $\lambda_{k}=\mu_{k}^{2}$ and the $\mu_{k}$ have the asymptotic representations

$$
\mu_{k}=k \frac{\pi}{a}+\tau_{0}+\frac{\tau_{1}}{k}+\frac{\tau_{2}}{k^{2}}+o\left(k^{-2}\right)
$$

and the numbers $\tau_{0}, \tau_{1}, \tau_{2}$ are as follows:

$$
\begin{gathered}
\tau_{0}=-\frac{2 \pi}{a}, \quad \tau_{1}=\frac{\left(1+\alpha^{2}\right) i}{\pi \alpha}+\frac{1}{4} \frac{G(a)}{\pi}, \\
\tau_{2}=\frac{2\left(1+\alpha^{2}\right) i}{\pi \alpha}-\frac{1}{2} \frac{a\left(1-\alpha^{2}\right)^{2}}{\pi^{2} \alpha^{2}}+\frac{1}{2} \frac{G(a)}{\pi} .
\end{gathered}
$$

In particular, the number of pure imaginary eigenvalues is even.

Remark 8. In [5], we have considered the differential equation (1) with the same boundary conditions $B_{3}, B_{4}$ at $a$ as in this paper but only one $\lambda$-dependent boundary conditions at 0 . We observe that the first two terms in the eigenvalue expansion coincide with those in Case 1 of [5], which differs from the present case that the $\lambda$-term is absent in the boundary condition (10). However, the third and fourth terms are similar but different.

\section{Acknowledgments}

Various of the above calculations have been verified with Sage. This work is based upon research supported by the National Research Foundation of South Africa under Grant number 80956. Any opinion, findings and conclusions or recommendations expressed in this material are those of the authors and therefore the NRF do not accept any liability in regard thereto.

\section{References}

[1] V. Pivovarchik and C. van der Mee, "The inverse generalized Regge problem," Inverse Problems, vol. 17, no. 6, pp. 1831-1845, 2001.

[2] M. Möller and V. Pivovarchik, "Spectral properties of a fourth order differential equation," Journal of Analysis and its Applications, vol. 25, no. 3, pp. 341-366, 2006.

[3] M. Möller and B. Zinsou, "Self-adjoint fourth order differential operators with eigenvalue parameter dependent boundary conditions," Quaestiones Mathematicae, vol. 34, no. 3, pp. 393-406, 2011.

[4] M. Möller and B. Zinsou, "Spectral asymptotics of self-adjoint fourth order differential operators with eigenvalue parameter dependent boundary conditions," Complex Analysis and Operator Theory, vol. 6, no. 3, pp. 799-818, 2012.

[5] M. Möller and B. Zinsou, "Spectral asymptotics of self-adjoint fourth order boundary value problem with eigenvalue parameter dependent boundary conditions," Boundary Value Problems, vol. 2012, article 106, 2012.

[6] Z. S. Aliyev and N. B. Kerimov, "Spectral properties of the differential operators of the fourth-order with eigenvalue parameter dependent boundary condition," International Journal of Mathematics and Mathematical Sciences, vol. 2012, Article ID 456517, 28 pages, 2012.

[7] N. B. Kerimov and Z. S. Aliev, "Basis properties of a spectral problem with a spectral parameter in the boundary condition," Matematicheskiu Sbornik, vol. 197, no. 10, pp. 65-86, 2006 (Russian), Translation in Sbornik: Mathematics, vol. 197, pp. 1467-1487, 2006.

[8] N. B. Kerimov and Z. S. Aliev, "On the basis property of the system of eigenfunctions of a spectral problem with a spectral parameter in the boundary condition," Differentsial'nye Uravneniya, vol. 43, no. 7, pp. 886-895, 2007 (Russian), Translation in Differential Equations, vol. 43, pp. 905-915, 2007.

[9] J. Weidmann, Spectral Theory of Ordinary Differential Operators, vol. 1258 of Lecture Notes in Mathematics, Springer, Berlin, Germany, 1987.

[10] R. Mennicken and M. Möller, Non-Self-Adjoint Boundary Eigenvalue Problems, vol. 192 of North-Holland Mathematics Studies, Elsevier, Amsterdam, The Netherlands, 2003. 


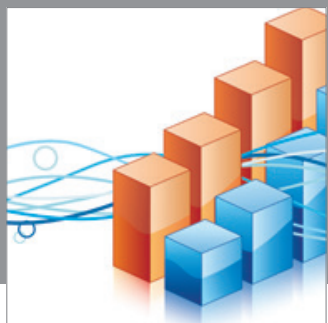

Advances in

Operations Research

mansans

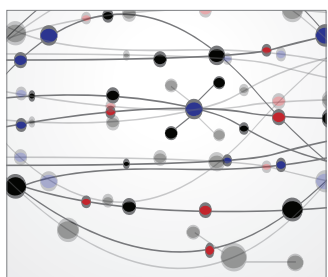

The Scientific World Journal
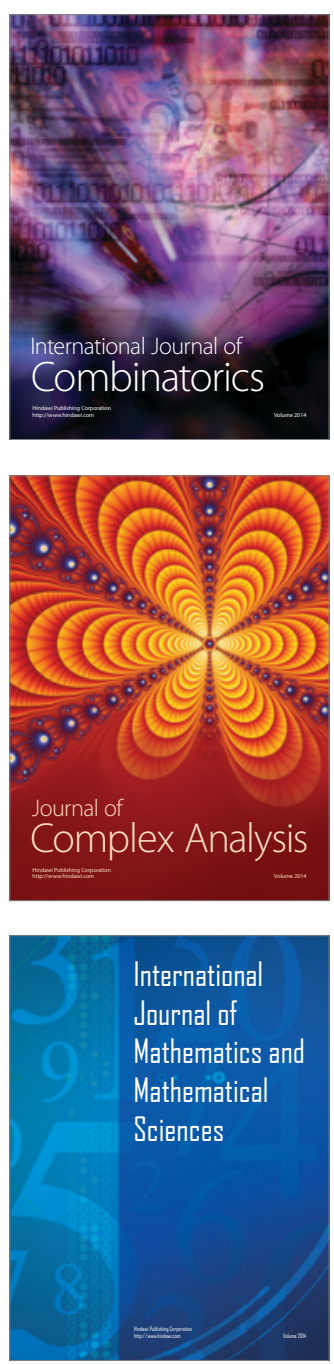
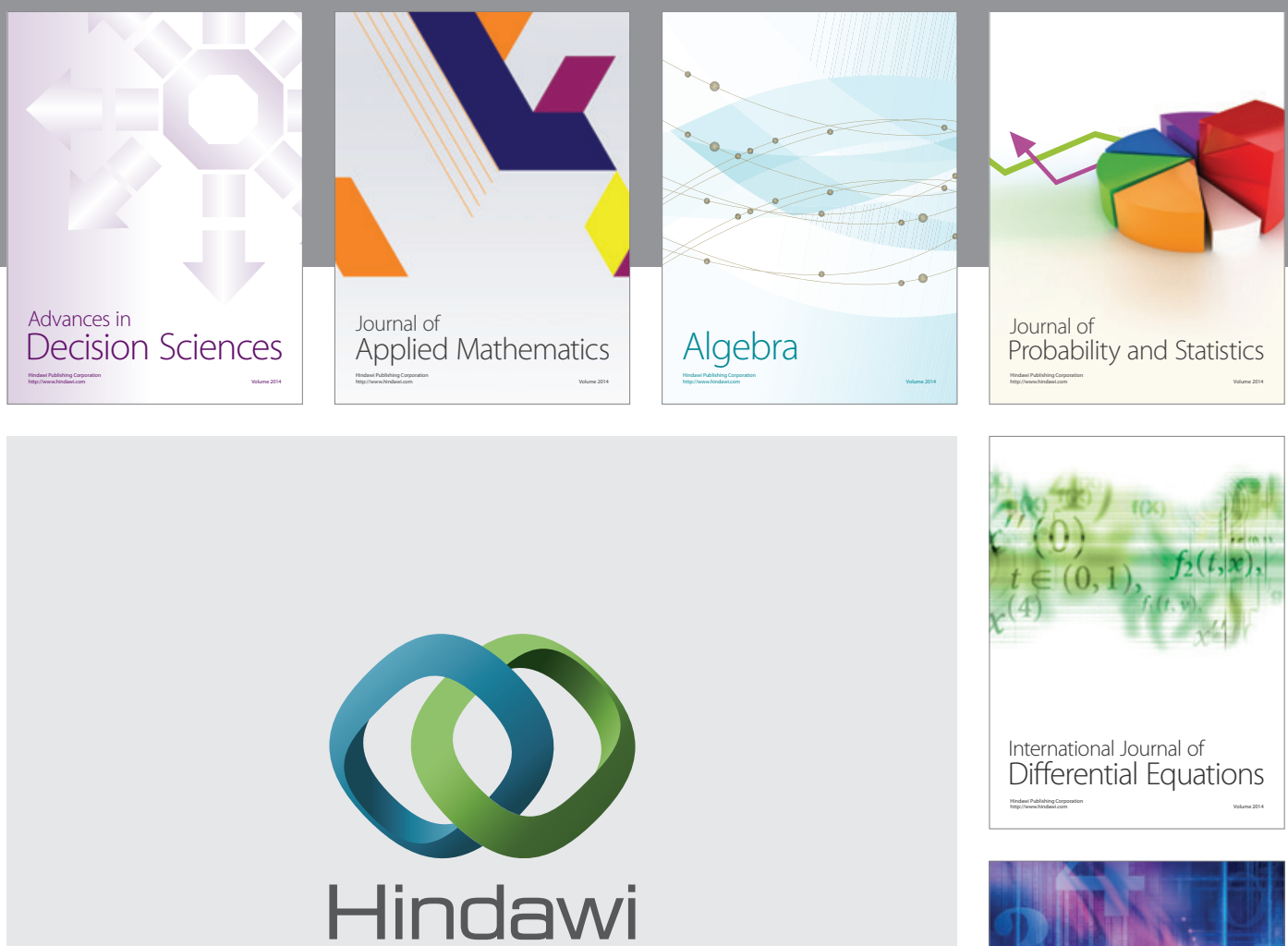

Submit your manuscripts at http://www.hindawi.com
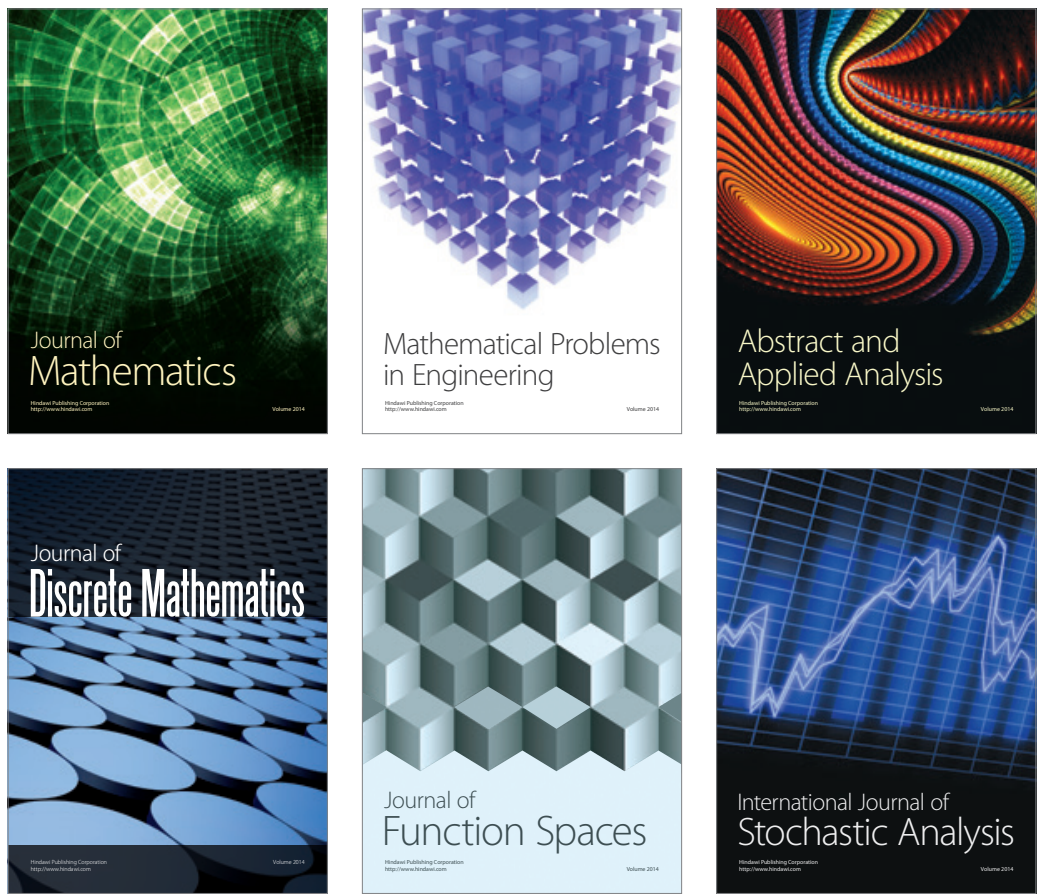

Journal of

Function Spaces

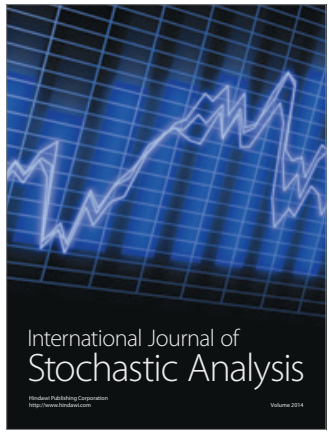

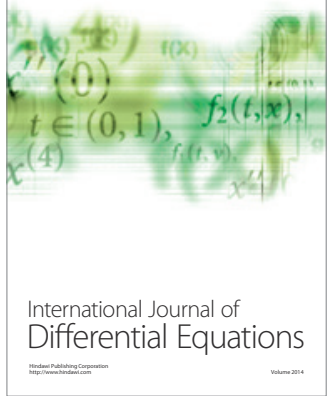
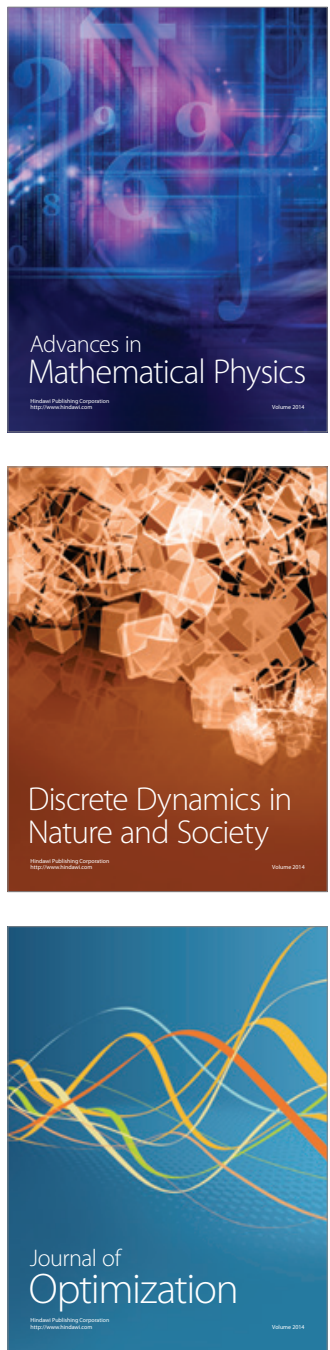